\title{
ENSAYOS DE GERMINACIÓN EN ENDEMISMOS IBÉRICOS
}

\author{
María Luisa CABELLO, Trinidad RUIZ y Juan Antonio DEVESA
}

\begin{abstract}
RESUMEN. Ensayos de germinación en endemismos ibéricos. Se han estudiado algunos aspectos relacionados con los factores que afectan la germinación en cinco endemismos ibéricos: Armeria linkiana Nieto Feliner, Digitalis mariana subsp. heywoodii P. \& M.Silva, Lavandula stoechas subsp. luisieri (Rozeira) Rozeira, Lavandula stoechas subsp. sampaiana Rozeira y Sanguisorba hybrida (L.)Nordborg. Se ha analizado el efecto del peróxido de hidrógeno, la acetona y el ácido sulfúrico como escarificantes químicos, así como la influencia de la luz en la germinación.
\end{abstract}

Palabras clave. Germinación, semilla, dormición, endemismo, Península Ibérica, Armeria linkiana, Digitalis mariana subsp. heywoodii, Lavandula stoechas subsp. luisieri, Lavandula stoechas subsp. sampaiana, Sanguisorba hybrida.

ABSTRACT. Germination assays in Iberian endemisms. We have studied some aspects related to factors that affect germination in five iberian endemisms: Armeria linkiana Nieto Feliner, Digitalis mariana subsp. heywoodii P. \& M.Silva, Lavandula stoechas subsp. luisieri (Rozeira) Rozeira, Lavandula stoechas subsp. sampaiana Rozeira and Sanguisorba hybrida (L.)Nordborg. We have analyzed the effect of hydrogen peroxide, acetone and sulphuric acid as chemical scarifiers, as well as the influence of light on germination.

Key words. Germination, seed, dormancy, endemism, Iberian Peninsula, Armeria linkiana, Digitalis mariana subsp. heywoodii, Lavandula stoechas subsp. luisieri, Lavandula stoechas subsp. sampaiana, Sanguisorba hybrida.

\section{INTRODUCCIÓN}

La germinación es el fenómeno conducente a la formación de una plántula a partir de una semilla, un proceso cuyo control se sabe está mediatizado por muchos factores. Requiere de un buen abastecimiento de agua, energía, una temperatura óptima, buena disponibilidad de oxígeno y una determinada acción de la luz (Bradbeer, 1988).

La germinación entraña en primer lugar una fase de imbibición, que depende de las características de la testa, después de la cual se establece rápidamente un flujo gaseoso entre el exterior y el interior de las semillas. Esta fase lleva consigo un notable incremento de la

Este trabajo ha sido financiado por la Dirección General de Investigación Científica y Técnica (DGICYT, PB90-0670) y la Consejería de Educación y Juventud (Junta de Extremadura)-Fondo Social Europeo (EIA94-13). 
actividad mitocondrial y está al igual que el resto del proceso germinativo muy influenciada por los factores térmico y lumínico (Mayer \& Shain, 1974).

Conocer cuales son las condiciones ambientales necesarias para que las semillas germinen es un aspecto de indudable utilidad práctica, de ahí que la información bibliográfica sea muy abundante en el terreno de la Agronomía, la Hortofruticultura y la Ingeniería forestal, si bien no puede decirse lo mismo en lo concerniente a las plantas silvestres, y mucho menos si éstas son endémicas. A nivel de la flora nacional, merece sin embargo especial mencion el trabajo de Mateo-Sagasta y Ceresuela Soria (1982), en el que se aportan datos acerca de la germinación de más de 300 taxones. Y en todo caso, este conocimiento es importante de cara a la conservación y gestión de los recursos vegetales, sobre todo a través de los Bancos de Semillas (Gómez Campo, 1979; Thompson, 1970).

En este contexto se ha planteado el presente trabajo, cuyo principal objetivo es dar a conocer algunos aspectos relacionados con los factores que afectan a la germinación

Armeria linkiana Nieto Feliner.

Badajoz: Tentudía. 8.VII.1996. J.A Devesa \& F. Vázquez. UNEX 21429

Digitalis mariana subsp. heywodii (P. Silva \& M. Silva) Hinz

Badajoz: Burguillos del Cerro. 8.VII.1996. J.A Devesa \& F. Vázquez. UNEX 21430

Lavandula stoechas subsp. luisieri (Rozeira) Rozeira Badajoz: Cruce de Fregenal de la Sierra a Cabeza la Vaca. 8.VII.1996. J.A Devesa \& F. Vázquez. UNEX 21431

Lavandula stoechas subsp. sampaiana Rozeira Badajoz: Badajoz, El Bote. 24.V.1996. J.A Devesa. UNEX 21432

Sanguisorba hybrida (L.) Nordborg Badajoz: Valle de Matamoros. 8.VII.1996. J.A Devesa \& F. Vázquez. UNEX 21433

Tabla 1. Procedencia del material estudiado. Provenance of the studied material. en cinco endemismos ibéricos: Armeria linkiana Nieto Feliner, Digitalis mariana subsp. heywoodii P. \& M.Silva, Lavandula stoechas subsp. luisieri (Rozeira) Rozeira, Lavandula stoechas subsp. sampaiana Rozeira y Sanguisorba hybrida (L.)Nordborg, taxones sobre los que no conocemos información publicada al respecto. En particular, con este trabajo se pretende estudiar el efecto de determinados agentes químicos y la luz sobre la germinación de semillas en los taxones seleccionados.

\section{MATERIAL Y MÉTODO}

\section{1.- Tests de germinación}

Durante Junio y Julio de 1996 se recolectaron semillas de individuos fructificados en poblaciones naturales, y pertenecientes a los siguientes taxones: Armeria linkiana, Digitalis mariana subsp. heywoodii, Lavandula stoechas subsp. luisieri, Lavandula stoechas subsp. sampaiana y Sanguisorba hybrida. (tab. 1).

Las semillas fueron depositadas en bolsas de papel etiquetadas y cerradas. Previamente se limpiaron de impurezas y elementos contaminantes, siendo inspeccionadas bajo observación estereoscópica (20 aumentos) al objeto de eliminar aquellas semillas sospechosas de inviabilidad. Posteriormente se conservaron durante 2-3 meses en condiciones de oscuridad y a temperatura ambiente del laboratorio $\left(22-28^{\circ} \mathrm{C}\right)$.

Los experimentos de germinación fueron llevados a cabo en un incubador REVCO (Modelo RI-50-555), en donde las semillas fueron colocadas en placas de Petri de $8.5 \mathrm{~cm}$ de diámetro, sobre un papel de filtro Albet humedecido a saturación con agua destilada. Se estudió una población por taxon, y 4 réplicas de 100 semillas por población, como sugiere la normativa de la ISTA (1985). Por el mismo 
motivo se eligió como duración de los experimentos un período de 30 días. Se consideraron germinadas aquellas semillas que habían originado una radícula de al menos 0.75 $\mathrm{mm}$ de longitud, efectuándose a diario el recuento y la retirada de las semillas germinadas.

Para evaluar la dormición se llevaron a cabo experimentos de germinación bajo condiciones controladas de temperatura, humedad relativa e iluminación, acordes con las recomendaciones de la ISTA (1985), así como con los registros publicados para las estaciones meteorológicas más cercanas (Jerez de los Caballeros y Badajoz-Base Aérea; Roldán Fernández, 1987). Así, las muestras de semillas utilizadas como control (C) fueron inducidas a germinar bajo una temperatura de $25^{\circ} \mathrm{C}$, una humedad relativa del $60 \%$ y un fotoperíodo en 7.5 horas bajo luz blanca fría, condiciones utilizadas también en las experiencias de escarificación, en las que previamente se habían sometido las semillas a la acción de algunos agentes químicos.

Experiencias similares, con escarificantes físicos y químicos que favorecen la germinación se conocen en especies próximas a las estudiadas, como por ejemplo: nitrato potásico en Armeria maritima (ISTA, 1985); acido succínico, ácido giberélico, sulfato de manganeso, molibdato amónico, rayos UV o altas temperaturas en Digitalis lanata (Ruminska \& Suchorska 1980); soluciones de pH ácido en Digitalis lanata (Ruminska et al. 1977), precalentamiento en Digitalis purpurea y Lavandula angustifolia (ISTA, 1985) y tratamiento con giberelinas en Lavandula angustifolia (ISTA, 1985).

Sin embargo, algunos de los productos químicos más comunes y que se mencionan en obras de referencia básicas (Rolston, 1978; Tran \& Cavanagh, 1984; Mayer \& PoljakoffMayber; 1982; Brabdeer 1988) no han sido experimentados en estos taxones. Entre estos pueden destacarse tres de conocida actividad erosionante sobre la cubierta seminal: acetona, peróxido de hidrógeno y ácido sulfúrico, agentes que han sido utilizados en el presente trabajo.

Los experimentos de escarificado químico realizados han sido los siguientes:

A: Inmersión de las semillas durante 5 minutos en acetona (99\%, Merck), con posterior lavado con abundante agua destilada e incubado en condiciones control.

H: Inmersión de las semillas durante 5 minutos en peróxido de hidrógeno ( $3 \%$, Sigma), con posterior lavado con abundante agua destilada e incubado en condiciones control.

S: Inmersión de las semillas durante 5 minutos en acido sulfúrico (96\%, Panreac), con posterior lavado con abundante agua destilada e incubado en condiciones control.

Para evaluar la influencia de la luz en dichos procesos, se repitieron éstos en oscuridad. Por tanto, se llevaron a cabo los siguientes:

Co: Condiciones control (C), pero sin iluminación alguna.

Ao: Condiciones del experimento $\mathbf{A}$, pero sin iluminación alguna.

Ho: Condiciones del experimento $\mathbf{H}$, pero sin iluminación alguna.

So: Condiciones del experimento $\mathbf{S}$, pero sin iluminación alguna.

\section{2.- Tratamiento y análisis de los datos}

Los datos obtenidos en las experiencias aportan información relativa a la capacidad germinativa de las muestras (germinación total al final de cada experimento, en $\%$ de germinación) y a la evolución del proceso germinativo a lo largo del período de estudio. Con estos últimos datos se construyeron curvas de germinación (fig. 1), y se calcularon los correspondientes valores del Iv. 
El Iv o índice de Vigor $(\operatorname{Iv}=(a / 1+b / 2+c /$ $3 \ldots .+\mathrm{z} / \mathrm{n}) \times 100 / \mathrm{s}$; donde $\mathrm{a}, \mathrm{b}, \mathrm{c}, \ldots \mathrm{z}$, es el número de semillas que germinan cada día; n el número de días que dura el experimento y s el número de semillas sembradas) es un parámetro muy útil a la hora de evaluar el proceso germinativo en términos de velocidad (Jain \& Saha, 1971). Su valor puede oscilar entre 0 y 100 , de manera que valores inferiores a 5 implican velocidades lentas; entre 5 y 11.11 medianas; entre 11.11 y 33.33 rápidas; y por encima de 33.33, muy rápidas (Brabdeer, 1988).

A los valores del porcentaje de germinación obtenidos para cada grupo de cuatro réplicas se les aplicó un test de homogeneidad de rangos a $\mathrm{p}=0.025$ (Test de Tolerancia de la ISTA, 1985), a fin de conocer si existían diferencias estadísticamente significativas entre los resultados obtenidos de las distintas réplicas para un mismo experimento y, por tanto, si parecía preferible la utilización de la media de dichos valores en cada 4 réplicas.

La dormición se cuantificó en términos de porcentaje de semillas no germinadas en cada experimento (Brabdeer, 1988). Para evaluar la acción de los distintos agentes químicos, se compararon los resultados del control (C) con los obtenidos para cada taxon en los experimentos $\mathbf{A}, \mathbf{H}$ y $\mathbf{S}$, aplicándoles el cálculo de los límites de significación del chi cuadrado para 1 grado de libertad, a un nivel de probabilidad del $5 \%$, para tests germinación en el que se utilicen 100 semillas, e incluida la corrección de Yates. Este método permite detectar -si las hubiere- las diferencias estadísticamente significativas a $p=0.05$, y se conoce como método estadístico de Roberts (vd. Brabdeer, 1988).

Finalmente, para evaluar la influencia de la luz, se compararon en cada taxon los resultados de los siguientes experimentos: $\mathbf{C}$ con $\mathbf{C o}, \mathbf{A}$ con Ao, $\mathbf{H}$ con $\mathbf{H o}$, y $\mathbf{S}$ con $\mathbf{S o}$, aplicándoles de nuevo el método de Roberts (1.c.).

\section{RESULTADOS Y DISCUSIÓN}

En los experimentos realizados, los porcentajes totales de germinación alcanzaron los valores que aparecen reflejados en la tabla 2. Al aplicar a dichos valores un test de homogeneidad de rangos a $\mathrm{p}=0.025$ (Test de Tolerancia de la ISTA, 1985) se encontró que las réplicas mostraban una variabilidad en la respuesta germinativa (tab. 3 ) que evidenciaba cierto grado de heterogeneidad en muestras estudiadas. Este hecho se atribuye no sólo a variaciones interindividuales, sino también intraindividuales, en función de la procedencia de cada fruto en términos reproductivos; consecuentemente, se estimó necesaria la utilización de los valores medios obtenidos para cada grupo de réplicas. La tabla 4, recoge los resultados de la aplicación del método de Roberts a dichos valores, y muestra los casos entre los que existen diferencias estadísticamente significativas $(p=0.05)$. Los valores de los índices de vigor para cada una de las muestras estudiadas se recogen en la tabla 2 .

Armeria linkiana Nieto Feliner, Anales Jard. Bot. Madrid 44: 332 (1987)

Hemicriptófito endémico del suroccidente peninsular, que aparece de manera natural en claros de matorral, sobre sustratos pedregosos y arenosos de carácter ácido, formando parte de fitocenosis

Tabla 2. Porcentajes totales de germinación e Índices de vigor (Iv, entre paréntesis) obtenidos en las diferentes réplicas de los experimentos control C y los escarificados con acetona $\mathbf{A}$, peróxido de hidrógeno $\mathbf{H}$, y sulfúrico $\mathbf{S}$, realizados con luz, así como sus homólogos ( $\mathbf{C o}, \mathbf{A o}, \mathbf{H o}$, y So), realizados en oscuridad. Total germination percentages and Vigor Indexes ( $I v$, in parenthesis) obtained for the different replicates of the $C$ control experiments and the scarifying treatments with acetone $A$, hydrogen peroxide $\boldsymbol{H}$, and sulphuric $\boldsymbol{S}$, carried out in light, or the named Co, Ao, Ho, and So, in darkness. 


\begin{tabular}{|c|c|c|c|c|c|c|c|c|c|}
\hline & & C & $\mathrm{C}_{0}$ & $\mathbf{A}$ & $\mathbf{A}_{0}$ & $\mathbf{H}$ & $\mathbf{H}_{0}$ & $\mathbf{S}$ & $\mathbf{S}_{0}$ \\
\hline \multirow[t]{5}{*}{ A. linkiana } & Réplica 1 & $\begin{array}{c}20 \\
(1.72)\end{array}$ & $\begin{array}{c}26 \\
(2.13)\end{array}$ & $\begin{array}{c}28 \\
(3.72)\end{array}$ & $\begin{array}{c}43 \\
(7.46)\end{array}$ & $\begin{array}{c}69 \\
(15.42)\end{array}$ & $\begin{array}{c}51 \\
(10.21)\end{array}$ & $\begin{array}{c}0 \\
(0.00)\end{array}$ & $\begin{array}{c}0 \\
(0.00)\end{array}$ \\
\hline & Réplica 2 & $\begin{array}{c}16 \\
(1.41)\end{array}$ & $\begin{array}{c}17 \\
(1.79)\end{array}$ & $\begin{array}{c}19 \\
(2.12)\end{array}$ & $\begin{array}{c}40 \\
(5.57)\end{array}$ & $\begin{array}{c}68 \\
(16.43)\end{array}$ & $\begin{array}{c}33 \\
(8.58)\end{array}$ & $\begin{array}{c}0 \\
(0.00)\end{array}$ & $\begin{array}{c}0 \\
(0.00)\end{array}$ \\
\hline & Réplica 3 & $\begin{array}{c}29 \\
(2.29)\end{array}$ & $\begin{array}{c}18 \\
(2.99)\end{array}$ & $\begin{array}{c}26 \\
(3.04)\end{array}$ & $\begin{array}{c}40 \\
(7.15)\end{array}$ & $\begin{array}{c}56 \\
(8.31)\end{array}$ & $\begin{array}{c}23 \\
(9.19)\end{array}$ & $\begin{array}{c}0 \\
(0.33)\end{array}$ & $\begin{array}{c}0 \\
(0.00)\end{array}$ \\
\hline & Réplica 4 & $\begin{array}{c}22 \\
(2.32)\end{array}$ & $\begin{array}{c}24 \\
(3.83)\end{array}$ & $\begin{array}{c}33 \\
(3.65)\end{array}$ & $\begin{array}{c}33 \\
(7.12)\end{array}$ & $\begin{array}{c}56 \\
(7.45)\end{array}$ & $\begin{array}{c}30 \\
(7.00)\end{array}$ & $\begin{array}{c}0 \\
(0.33)\end{array}$ & $\begin{array}{c}0 \\
(0.00)\end{array}$ \\
\hline & Media & $\begin{array}{l}21.75 \\
(1.94)\end{array}$ & $\begin{array}{l}21.25 \\
(2.69)\end{array}$ & $\begin{array}{l}26.50 \\
(3.13)\end{array}$ & $\begin{array}{l}39.00 \\
(6.82)\end{array}$ & $\begin{array}{c}62.25 \\
(11.90)\end{array}$ & $\begin{array}{l}34.25 \\
(8.75)\end{array}$ & $\begin{array}{c}0.00 \\
(0.165)\end{array}$ & $\begin{array}{c}0.00 \\
(0.00)\end{array}$ \\
\hline \multirow[t]{5}{*}{ D. mariana subsp. heywoodii } & Réplica 1 & $\begin{array}{c}54 \\
(7.61)\end{array}$ & $\begin{array}{c}91 \\
(3.69)\end{array}$ & $\begin{array}{c}83 \\
(11.11)\end{array}$ & $\begin{array}{c}77 \\
(8.27)\end{array}$ & $\begin{array}{c}53 \\
(7.24)\end{array}$ & $\begin{array}{c}46 \\
(4.42)\end{array}$ & $\begin{array}{c}0 \\
(0.00)\end{array}$ & $\begin{array}{c}0 \\
(0.00)\end{array}$ \\
\hline & Réplica 2 & $\begin{array}{c}62 \\
(8.61)\end{array}$ & $\begin{array}{c}79 \\
(0.98)\end{array}$ & $\begin{array}{c}89 \\
(11.94)\end{array}$ & $\begin{array}{c}81 \\
(9.06)\end{array}$ & $\begin{array}{c}57 \\
(7.04)\end{array}$ & $\begin{array}{c}25 \\
(2.80)\end{array}$ & $\begin{array}{c}0 \\
(0.00)\end{array}$ & $\begin{array}{c}0 \\
(0.00)\end{array}$ \\
\hline & Réplica 3 & $\begin{array}{c}74 \\
(10.80)\end{array}$ & $\begin{array}{c}98 \\
(0.07)\end{array}$ & $\begin{array}{c}73 \\
(10.89)\end{array}$ & $\begin{array}{c}63 \\
(7.14)\end{array}$ & $\begin{array}{c}59 \\
(9.30)\end{array}$ & $\begin{array}{c}28 \\
(2.96)\end{array}$ & $\begin{array}{c}0 \\
(0.00)\end{array}$ & $\begin{array}{c}0 \\
(0.00)\end{array}$ \\
\hline & Réplica 4 & $\begin{array}{c}61 \\
(9.64)\end{array}$ & $\begin{array}{c}88 \\
(0.72)\end{array}$ & $\begin{array}{c}71 \\
(7.60)\end{array}$ & $\begin{array}{c}65 \\
(6.32)\end{array}$ & $\begin{array}{c}28 \\
(3.74)\end{array}$ & $\begin{array}{c}29 \\
(3.08)\end{array}$ & $\begin{array}{c}0 \\
(0.00)\end{array}$ & $\begin{array}{c}0 \\
(0.00)\end{array}$ \\
\hline & Media & $\begin{array}{l}62.75 \\
(9.17)\end{array}$ & $\begin{array}{l}89.00 \\
(1.37)\end{array}$ & $\begin{array}{c}79.00 \\
(10.39)\end{array}$ & $\begin{array}{l}71.50 \\
(7.70)\end{array}$ & $\begin{array}{l}49.25 \\
(6.83)\end{array}$ & $\begin{array}{l}32.00 \\
(3.32)\end{array}$ & $\begin{array}{c}0.00 \\
(0.00)\end{array}$ & $\begin{array}{c}0.00 \\
(0.00)\end{array}$ \\
\hline \multirow[t]{5}{*}{ L. stoechas subsp. luisieri } & Réplica 1 & $\begin{array}{c}51 \\
(2.40)\end{array}$ & $\begin{array}{c}3 \\
(0.44)\end{array}$ & $\begin{array}{c}67 \\
(4.19)\end{array}$ & $\begin{array}{c}69 \\
(6.22)\end{array}$ & $\begin{array}{c}77 \\
(5.50)\end{array}$ & $\begin{array}{c}43 \\
(2.54)\end{array}$ & $\begin{array}{c}75 \\
(6.13)\end{array}$ & $\begin{array}{c}35 \\
(3.14)\end{array}$ \\
\hline & Réplica 2 & $\begin{array}{c}46 \\
(2.49)\end{array}$ & $\begin{array}{c}8 \\
(0.12)\end{array}$ & $\begin{array}{c}74 \\
(4.37)\end{array}$ & $\begin{array}{c}63 \\
(6.43)\end{array}$ & $\begin{array}{c}98 \\
(6.32)\end{array}$ & $\begin{array}{c}34 \\
(1.99)\end{array}$ & $\begin{array}{c}93 \\
(7.90)\end{array}$ & $\begin{array}{c}32 \\
(2.32)\end{array}$ \\
\hline & Réplica 3 & $\begin{array}{c}49 \\
(2.33)\end{array}$ & $\begin{array}{c}5 \\
(0.14)\end{array}$ & $\begin{array}{c}70 \\
(3.98)\end{array}$ & $\begin{array}{c}54 \\
(5.31)\end{array}$ & $\begin{array}{c}80 \\
(5.19)\end{array}$ & $\begin{array}{c}45 \\
(2.53)\end{array}$ & $\begin{array}{c}78 \\
(6.14)\end{array}$ & $\begin{array}{c}22 \\
(2.05)\end{array}$ \\
\hline & Réplica 4 & $\begin{array}{c}54 \\
(2.62)\end{array}$ & $\begin{array}{c}5 \\
(0.44)\end{array}$ & $\begin{array}{c}76 \\
(4.76)\end{array}$ & $\begin{array}{c}65 \\
(6.71)\end{array}$ & $\begin{array}{c}78 \\
(5.04)\end{array}$ & $\begin{array}{c}32 \\
(1.62)\end{array}$ & $\begin{array}{c}86 \\
(6.23)\end{array}$ & $\begin{array}{c}31 \\
(2.56)\end{array}$ \\
\hline & Media & $\begin{array}{l}50.00 \\
(2.46)\end{array}$ & $\begin{array}{c}5.25 \\
(0.28)\end{array}$ & $\begin{array}{l}71.75 \\
(4.33)\end{array}$ & $\begin{array}{l}62.75 \\
(6.17)\end{array}$ & $\begin{array}{l}83.25 \\
(5.51)\end{array}$ & $\begin{array}{l}38.50 \\
(2.17)\end{array}$ & $\begin{array}{l}83.00 \\
(6.60)\end{array}$ & $\begin{array}{l}30.00 \\
(2.52)\end{array}$ \\
\hline \multirow[t]{5}{*}{ L. stoechas subsp. sampaiana } & Réplica 1 & $\begin{array}{c}18 \\
(1.51)\end{array}$ & $\begin{array}{c}3 \\
(2.91)\end{array}$ & $\begin{array}{c}18 \\
(2.28)\end{array}$ & $\begin{array}{c}18 \\
(2.09)\end{array}$ & $\begin{array}{c}29 \\
(3.59)\end{array}$ & $\begin{array}{c}9 \\
(1.04)\end{array}$ & $\begin{array}{c}40 \\
(5.42)\end{array}$ & $\begin{array}{c}20 \\
(3.12)\end{array}$ \\
\hline & Réplica 2 & $\begin{array}{c}28 \\
(2.30)\end{array}$ & $\begin{array}{c}2 \\
(4.14)\end{array}$ & $\begin{array}{c}18 \\
(1.76)\end{array}$ & $\begin{array}{c}15 \\
(2.17)\end{array}$ & $\begin{array}{c}20 \\
(3.04)\end{array}$ & $\begin{array}{c}3 \\
(0.18)\end{array}$ & $\begin{array}{c}48 \\
(8.47)\end{array}$ & $\begin{array}{c}19 \\
(3.08)\end{array}$ \\
\hline & Réplica 3 & $\begin{array}{c}28 \\
(2.88)\end{array}$ & $\begin{array}{c}3 \\
(4.42)\end{array}$ & $\begin{array}{c}12 \\
(0.75)\end{array}$ & $\begin{array}{c}13 \\
(2.26)\end{array}$ & $\begin{array}{c}22 \\
(2.86)\end{array}$ & $\begin{array}{c}3 \\
(0.87)\end{array}$ & $\begin{array}{c}47 \\
(5.62)\end{array}$ & $\begin{array}{c}14 \\
(2.40)\end{array}$ \\
\hline & Réplica 4 & $\begin{array}{c}33 \\
(3.24)\end{array}$ & $\begin{array}{c}4 \\
(2.65)\end{array}$ & $\begin{array}{c}19 \\
(1.39)\end{array}$ & $\begin{array}{c}11 \\
(1.83)\end{array}$ & $\begin{array}{c}23 \\
(2.52)\end{array}$ & $\begin{array}{c}4 \\
(0.59)\end{array}$ & $\begin{array}{c}42 \\
(6.37)\end{array}$ & $\begin{array}{c}14 \\
(2.26)\end{array}$ \\
\hline & Media & $\begin{array}{l}26.75 \\
(2.48)\end{array}$ & $\begin{array}{c}3.00 \\
(0.29)\end{array}$ & $\begin{array}{l}16.75 \\
(1.55)\end{array}$ & $\begin{array}{l}14.25 \\
(2.10)\end{array}$ & $\begin{array}{l}23.50 \\
(3.00)\end{array}$ & $\begin{array}{c}4.75 \\
(0.67)\end{array}$ & $\begin{array}{l}44.25 \\
(6.47)\end{array}$ & $\begin{array}{l}16.75 \\
(2.72)\end{array}$ \\
\hline \multirow[t]{5}{*}{ S. hybrida } & Réplica 1 & $\begin{array}{c}82 \\
(8.65)\end{array}$ & $\begin{array}{c}26 \\
(2.91)\end{array}$ & $\begin{array}{c}46 \\
(2.99)\end{array}$ & $\begin{array}{c}56 \\
(4.84)\end{array}$ & $\begin{array}{c}86 \\
(8.47)\end{array}$ & $\begin{array}{c}14 \\
(1.50)\end{array}$ & $\begin{array}{c}98 \\
(10.90)\end{array}$ & $\begin{array}{c}23 \\
(3.01)\end{array}$ \\
\hline & Réplica 2 & $\begin{array}{c}88 \\
(9.12)\end{array}$ & $\begin{array}{c}36 \\
(4.14)\end{array}$ & $\begin{array}{c}44 \\
(2.45)\end{array}$ & $\begin{array}{c}40 \\
(3.30)\end{array}$ & $\begin{array}{c}63 \\
(6.10)\end{array}$ & $\begin{array}{c}24 \\
(2.95)\end{array}$ & $\begin{array}{c}90 \\
(10.30)\end{array}$ & $\begin{array}{c}25 \\
(3.25)\end{array}$ \\
\hline & Réplica 3 & $\begin{array}{c}80 \\
(7.94)\end{array}$ & $\begin{array}{c}37 \\
(4.42)\end{array}$ & $\begin{array}{c}49 \\
(3.10)\end{array}$ & $\begin{array}{c}48 \\
(4.31)\end{array}$ & $\begin{array}{c}80 \\
(8.70)\end{array}$ & $\begin{array}{c}20 \\
(2.34)\end{array}$ & $\begin{array}{c}93 \\
(12.05)\end{array}$ & $\begin{array}{c}28 \\
(3.81)\end{array}$ \\
\hline & Réplica 4 & $\begin{array}{c}89 \\
(7.64)\end{array}$ & $\begin{array}{c}21 \\
(2.65)\end{array}$ & $\begin{array}{c}61 \\
(3.62)\end{array}$ & $\begin{array}{c}37 \\
(3.16)\end{array}$ & $\begin{array}{c}84 \\
(9.57)\end{array}$ & $\begin{array}{c}20 \\
(2.18)\end{array}$ & $\begin{array}{c}89 \\
(12.08)\end{array}$ & $\begin{array}{c}20 \\
(2.53)\end{array}$ \\
\hline & Media & $\begin{array}{l}84.75 \\
(8.34)\end{array}$ & $\begin{array}{l}30.00 \\
(3.53)\end{array}$ & $\begin{array}{l}50.00 \\
(3.04)\end{array}$ & $\begin{array}{l}45.25 \\
(3.90)\end{array}$ & $\begin{array}{l}78.25 \\
(8.21)\end{array}$ & $\begin{array}{l}19.50 \\
(2.24)\end{array}$ & $\begin{array}{c}92.50 \\
(11.33)\end{array}$ & $\begin{array}{l}24.00 \\
(3.15)\end{array}$ \\
\hline
\end{tabular}


dominadas por elementos graminoides vivaces (Agrostio-Stipion giganteae, Lygeo-Stipetea). Presenta semillas de 0.9-1 × $2.2 .5 \mathrm{~mm}$, que alberga en cápsulas monospermas con pericarpo membranoso que se abre de modo circunciso o irregular (Devesa, 1995; Nieto. Feliner, 1990).

En las muestras estudiadas las semillas alcanzaron un porcentaje de germinación del 21.75 $\%$ en las condiciones control (C), es decir, un nivel de dormición del $78.25 \%$, fenómeno que ya había sido constatado experimentalmente en otras especies del género (vg. Armeria maritima; Hutchings \& Russel, 1989).

La aplicación de acetona como escarificante químico (A) no incrementó significativamente el porcentaje de germinación, algo que sí se observó al aplicar peróxido de hidrógeno $(\mathbf{H})$, alcanzándose entonces niveles germinativos del $62.25 \%$, lo que tal vez pueda ser debido a que el oxidante ejerza una acción que favorezca la entrada de agua en la semilla, e incluso evitar la contaminación por hongos y bacterias (Tran \& Cavanagh, 1984; Pahl, 1993). Por el contrario, el ácido sulfúrico (S), cuya acción sobre la testa seminal ha sido probada en muy diversas familias (Pérez et al., 1995; Tischler et al., 1994), impidió totalmente el proceso germinativo, probablemente por tratarse de un agente excesivamente agresivo para estas semillas.

En relación con la respuesta a la luz, indicar que sólo en el caso de los experimentos efectuados con peróxido de hidrógeno $(\mathbf{H})$ se apreció un comportamiento fotoblástico, siendo éste positivo, al igual que sucede en Armeria maritima subsp. intermedia (Ryttari \& Lahti, 1992).

En relación con los índices de vigor, señalar que los más altos fueron los de los experimentos $\mathbf{H}$, en donde alcanzaron un valor igual a 11.90. Dicho valor correspondería al caso teórico en que todas las semillas de una réplica germinasen el noveno día de experimentación, lo cual estaría relacionado con una velocidad de germinación mediana. Por eso en este caso el tratamiento de elección a proponer sería el $\mathbf{H}$.

Digitalis mariana subsp. heywodii P. \& M.Silva, Agron. Lusit. 20: 239 (1959)

Hemicriptófito característico del sector mariánico-monchiquense de la provincia luso- extremadurense, que aparece en enclaves pedregosos, tanto graníticos como pizarrosos, formando parte de comunidades subrupícolas (Rumici-Dianthion, Asplenietea). Sus semillas, de 0.5-0.9 x 0.4-0.6 mm y coloración pardusca, proceden de cápsulas polispermas, y presentan una morfología prismática o trígona, con un surco característico en la cara ventral (Devesa, 1995; Juan Rodríguez, 1994).

La muestra de semillas estudiadas presentó en condiciones control (C) una tasa germinativa del $62.75 \%$, o sea, una dormición del $37.25 \%$, fenómeno que era conocido en el género (Shchelokova, 1976; Woodward \& Jones, 1984). La aplicación de acetona como agente escarificante no produjo incrementos significativos de este porcentaje. Por el contrario, resultó negativa la acción de los otros dos productos químicos utilizados, tanto el peróxido $(\mathbf{H})$, que rebajó significativamente la germinación (a un 49.25 $\%)$ como el sulfúrico (S), que la impidió completamente. En ambos casos se interpreta que la acción de los agentes químicos utilizados sobre la testa produjo un deterioro excesivo de la misma y, posiblemente, daños al embrión.

Por otra parte, el tratamiento con acetona (A) propició valores del Iv de 10.39 , que se relacionan con una velocidad germinativa de décimo día, algo superior a la obtenida en condiciones control (9.17: de undécimo día), una velocidad claramente mayor que la obsevada con los restantes experimentos, por lo que el tratamiento (A) resultó el más adecuado para este taxon.

En relación con la influencia de la luz, destacar que este taxon ha mostrado una fotoblastia diferente según los casos. Así, en los experimentos control el comportamiento ha sido fotoblástico negativo, en los de acetona indiferente y en los de peróxido de hidrógeno fotoblástico positivo (las comparaciones en el caso del sulfúrico no pudieron hacerse por falta de germinación), fenómeno este último que había sido previamente señalado en Digitalis purpurea (Thompson, 1989). La diversidad de resultados obtenidos ilustra acerca de la multiplicidad de factores que condicionan el inicio del proceso germinativo. Dada la relativa rareza de fotoblastia negativa en la naturaleza (Brabdeer, 1988), los resultados del control tienen un especial interés, y posiblemente estén relacionados con el microhábitat esciáfilo que existe en las fisuras de roca donde vive esta planta de manera natural. 


\begin{tabular}{lcccccccc}
\hline & $\mathbf{C}$ & $\mathbf{C}_{\mathbf{0}}$ & $\mathbf{A}$ & $\mathbf{A}_{\mathbf{0}}$ & $\mathbf{H}$ & $\mathbf{H}_{\mathbf{0}}$ & $\mathbf{S}$ & $\mathbf{S}_{\mathbf{0}}$ \\
\hline $\begin{array}{l}\text { A. linkiana } \\
\begin{array}{l}\text { D. marianasubsp. } \\
\text { heywodii }\end{array}\end{array}$ & $*$ & $*$ & $*$ & $*$ & $*$ & $*$ & $\mathrm{sd}$ & $\mathrm{sd}$ \\
$\begin{array}{l}\text { L. stoechas subsp. } \\
\text { luisieri }\end{array}$ & $\mathrm{ns}$ & $\mathrm{ns}$ & $\mathrm{ns}$ & $\mathrm{ns}$ & $*$ & $\mathrm{~ns}$ & $*$ & $\mathrm{~ns}$ \\
$\begin{array}{l}\text { L. stoechas subsp. } \\
\text { sampaiana }\end{array}$ & $\mathrm{ns}$ & $\mathrm{ns}$ & $\mathrm{ns}$ & $\mathrm{ns}$ & $\mathrm{ns}$ & $\mathrm{ns}$ & $\mathrm{ns}$ & $\mathrm{ns}$ \\
\begin{tabular}{l} 
S. hybrida \\
\hline
\end{tabular} & $\mathrm{ns}$ & $\mathrm{ns}$ & $\mathrm{ns}$ & $\mathrm{ns}$ & $*$ & $\mathrm{~ns}$ & $\mathrm{~ns}$ & $\mathrm{~ns}$ \\
\hline
\end{tabular}

Tabla 3. Resultados obtenidos tras la aplicación del Test de Tolerancia (ISTA, 1985), para poner de manifiesto las diferencias estadísticamente significativas $(\mathrm{p}=0.025)$ entre los porcentajes obtenidos en las distintas réplicas de un mismo experimento: $\mathbf{C}$, experimentos control; $\mathbf{A}, \mathbf{H}, \mathbf{S}$, escarificados con acetona, peróxido de hidrógeno y sulfúrico, todos ellos, realizados con luz. Sus homólogos: Co, Ao, Ho, y So realizados en oscuridad. $*=$ existen diferencias, $\mathrm{ns}=$ no existen, $\mathrm{sd}=$ sin datos. Results obtained when the Tolerance Test (ISTA, 1985), is applied. It is used to show the statistically significant differences $(p=0.025)$ among the replicates of each experiment: $\boldsymbol{C}$ control experiments; $\boldsymbol{A}, \boldsymbol{H}, \boldsymbol{S}$, scarified with acetone, hydrogen peroxide and sulphuric, all them carried out in light. The named $\mathbf{C o}, \mathbf{A o}, \mathbf{H o}$, and So, in darkness. * = there are differences, $n s=$ there are not, sd=no data available.

Lavandula stoechas subsp. luisieri (Rozeira) Rozeira, Agron. Lusit. 24: 173 (1964)

Caméfito silicícola del occidente peninsular, que aparece frecuentemente ligado a enclaves mediterráneos con cierto carácter oceánico. Por ello es elemento característico de los matorrales de sustitución de alcornocales tanto mesomediterráneos (Sanguisorbo-Querceto suberis S.) como termomediterráneos (Oleo-Querceto suberis $S$, Myrto-Querceto suberis S.). Posee frutos tetranúcula, de color pardusco, rugosas y glabras, de 0.9 -1 x 1-2 mm (Devesa 1987a, 1995; Rivas Martínez 1987; Rivas Martínez \& al. 1990).

Al sembrar las núculas en condiciones control (C) se obtuvieron valores de germinación del $50 \%$. Se tenían datos acerca de la existencia de dormición a nivel de género (Bernal \& Torres Cases, 1997; Iriondo et al., 1992; Chavagnat, 1978), y de cómo el escarificado había incrementado la tasa germinativa en otros representantes del mismo, bien a través del peróxido de hidrógeno (Alvarez Tinaut et al., 1968) como de la acción de ácidos (Singh \& Srivastava, 1990), si bien hay que matizar que Cobo \& Andreu (1988) demostraron lo contrario con los ácidos del tramo digestivo de la tortuga mora.

La acción de escarificantes sobre las núculas aumentó significativamente los porcentajes de germinacion en el material estudiado, alcanzándose el $71.75 \%$ con la acetona (A), el $83.25 \%$ con el peróxido (H) y el $83 \%$ con el ácido sulfúrico (S). Estos resultados son coherentes con el hecho de que la testa seminal está cubierta por el pericarpo del fruto, lo que implica una mayor necesidad de que se favorezca la eliminación de estas estructuras para facilitar la germinación.

Por otra parte, resulta curioso comparar estos resultados tan positivos en términos porcentuales con los Iv correspondientes pues, como puede observarse en la tabla 2 , se trata de valores inferiores a $I v=6.60$, que implican velocidades medianas $O$ lentas, lo cual está mostrando indirectamente que aquí, el proceso germinativo sigue un modelo cinéticamente diferente. Esto es evidente sobre todo si se comparan las curvas, que corresponden a una germinación mucho más gradual en el tiempo.

Respecto al comportamiento en relación con la luz, es destacable la fotoblastia positiva encontrada en todos los casos, salvo las núculas escarificadas con acetona. Este comportamiento 
a

\begin{tabular}{|c|c|c|c|}
\hline & $\mathrm{C} / \mathrm{A}$ & $\mathrm{C} / \mathrm{H}$ & $\mathrm{C} / \mathrm{S}$ \\
\hline A. linkiana & ns & * & $*$ \\
\hline $\begin{array}{l}\text { D. mariana subsp. } \\
\text { heywodii }\end{array}$ & $*$ & ns & $*$ \\
\hline $\begin{array}{l}\text { L. stoechas subsp. } \\
\text { luisieri }\end{array}$ & * & $*$ & $*$ \\
\hline $\begin{array}{l}\text { L. stoechas subsp. } \\
\text { sampaiana }\end{array}$ & $\mathrm{ns}$ & $\mathrm{ns}$ & $*$ \\
\hline S. hybrida & $*$ & ns & ns \\
\hline
\end{tabular}

b

\begin{tabular}{lcccc}
\hline & $\mathbf{C} / \mathbf{C}_{\mathbf{0}}$ & $\mathbf{A} / \mathbf{A}_{\mathbf{0}}$ & $\mathbf{H} / \mathbf{H}_{0}$ & $\mathbf{S} / \mathbf{S}_{\mathbf{0}}$ \\
\hline $\begin{array}{l}\text { A. linkiana } \\
\text { De mariana subsp. }\end{array}$ & $*$ & $\mathrm{~ns}$ & $*$ & - \\
$\begin{array}{l}\text { heywodii } \\
\text { L. stoechas subsp. }\end{array}$ & $*$ & $\mathrm{~ns}$ & $*$ & $*$ \\
$\begin{array}{l}\text { luisieri } \\
\text { L. stoechas subsp. } \\
\text { sampaiana }\end{array}$ & $*$ & $\mathrm{~ns}$ & $*$ & $*$ \\
\begin{tabular}{l} 
S. hybrida \\
\hline
\end{tabular} & $*$ & $\mathrm{~ns}$ & $*$ & $*$ \\
\hline
\end{tabular}

Tabla 4 (a y b). Resultados obtenidos tras la aplicación del cálculo de los límites de significación del chi cuadrado para 1 grado de libertad, a un nivel de probabilidad del $5 \%$ (método de Roberts, in Brabdeer 1988), para evaluar las diferencias estadísticamente significativas entre (a) el control C y los escarificados con acetona $\mathbf{A}$, peróxido de hidrógeno $\mathbf{H}$, y sulfúrico $\mathbf{S}$ y (b) entre los experimentos realizados con luz, (C, $\mathbf{A}, \mathbf{H}$, y S) y éstos mismos en oscuridad $(\mathbf{C o}, \mathbf{A o}, \mathbf{H o}$, y So $){ }^{*}=$ existen diferencias, ns = no existen. Results obtained when we calculate the limits of significance of the chi squared for one degree of freedom, at a 5 $\%$ probability level (method of Roberts, in Brabdeer, 1988). It evaluates the statistically significant differences $(p=0.025)$ between $(\boldsymbol{a})$ the control treatment $\boldsymbol{C}$ and the scarification with acetone $A$, hydrogen peroxide $\boldsymbol{H}$ and sulphuric $\boldsymbol{S}$ and $(\boldsymbol{b})$ between the experiments carried out in light $(\boldsymbol{C}, \boldsymbol{A}, \boldsymbol{H}$, and $\boldsymbol{S})$ and in darkness $(\mathrm{Co}, \mathbf{A o}, \mathrm{Ho}$, y So $) . *^{*}$ there are differences, $n s=$ there are not.

puede estar justificado por la especial adaptación que tiene el taxon a vivir de manera natural en los ambientes heliófilos de los matorrales esclerófilos mediterráneos.

Lavandula stoechas subsp. sampaiana Rozeira, Brotéria (Ser. Ci. Nat.) 18: 70 (1949)

Caméfito muy próximo al anterior desde el punto de vista taxonómico, pero que tiene una corología más amplia, pues se extiende por buena parte del territorio occidental ibérico, así como una ecología más ligada a las series del encinar, en los pisos termomediterráneo y mesomediterráneo (Pyro - Querceto rotundifoliae S., Myrto - Querceto rotundifoliae S.), siempre sobre sustratos ácidos. Posee frutos tetranúcula, de caracteres muy similares a la subespecie anterior (Devesa, 1987a, 1995; Rivas Martínez, 1987).

En este caso, las núculas sembradas bajo condiciones control (C) germinaron en un $26.75 \%$, es decir, en un porcentaje significativamente menor a como lo hicieron las de la subsp. luisieri. Si a esto se añade que ni el pretratamiento con acetona (A) ni con peróxido $(\mathbf{H})$ incrementaron significativamente este porcentaje, puede concluirse que ambas subespecies han mostrado un comportamiento germinativo distinto. Ello pone en evidencia el interés del estudio de los requerimientos germinativos de cada taxon. Por contra, la adición de sulfúrico (S) sí incrementó significativamente los porcentajes germinativos, concretamente hasta niveles del $44.25 \%$.

$\mathrm{Al}$ analizar los valores de Iv encontrados se observa, además, que el proceso tiene velocidades lentas en todos los casos, salvo tras la escarificación con sulfúrico (S), tratamiento que propició una velocidad germinativa mediana, siendo pues el tratamiento más eficaz en este taxon.

En relación con la luz, los resultados encontrados en este taxon siguen una pauta idéntica a la de la subespecie anterior, lo que puede obedecer a las mismas causas.

Sanguisorba hybrida (L.) Nordborg, Op. Bot. (Lund) 11 (2): 67 (1966) 

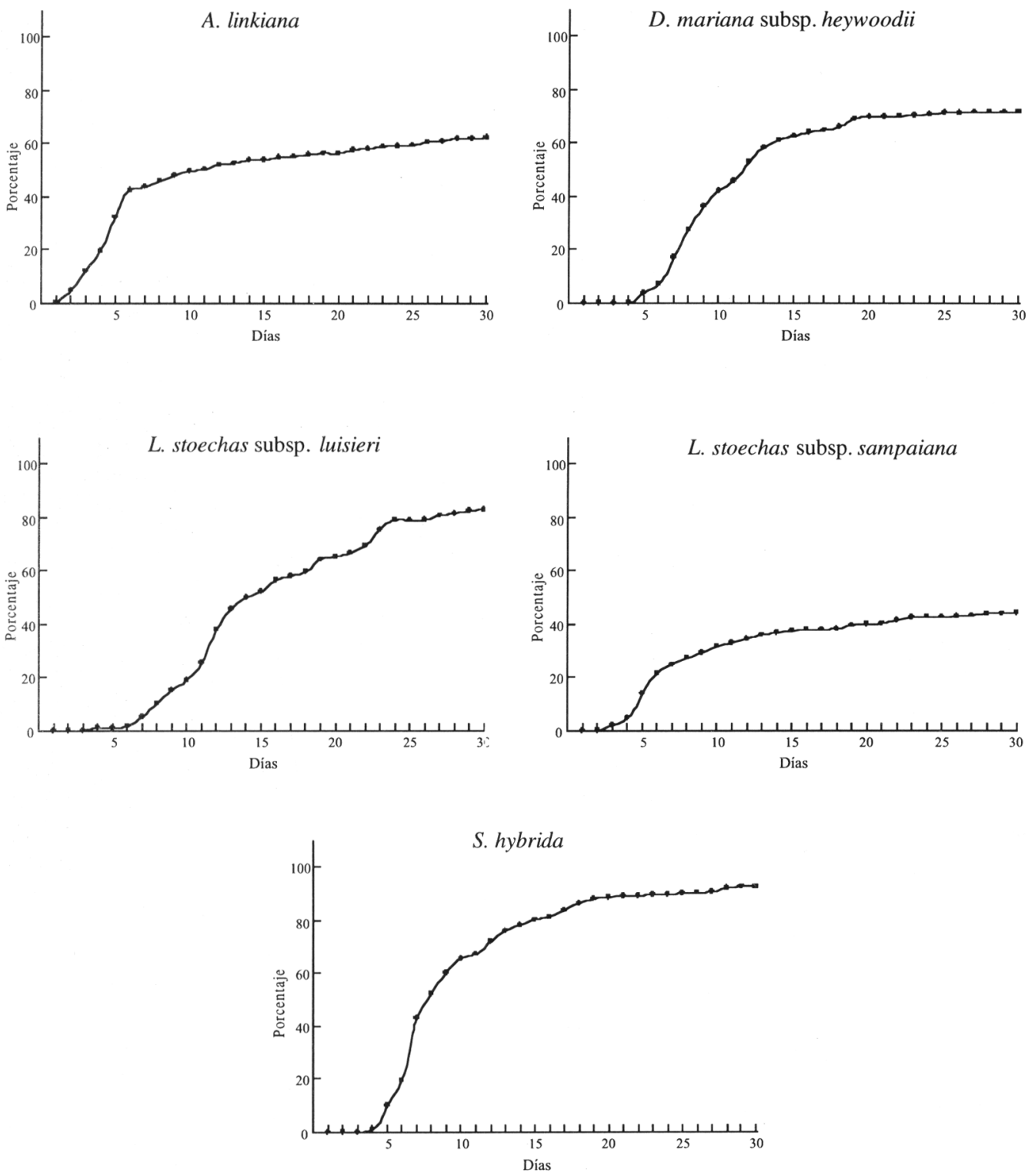

Figura 1. Porcentajes acumulados de germinación obtenidos a lo largo de un periodo experimental de 30 días, en los taxones estudiados. En cada taxon, se han representado los resultados obtenidos con el escarificado que ha resultado más favorable: peróxido de hidrógeno y luz (H ) para Armeria linkiana, acetona y luz (A) para Digitalis mariana subsp. heywoodii, y sulfúrico y luz (S) para Lavandula stoechas subsp. luisieri, Lavandula stoechas subsp. sampaiana y Sanguisorba hybrida. Cumulative percentages of germination obtained for a 30 days experimental period, in the studied taxa. For each taxon, we have represented the results obtained with the most favourable scarifying method: hydrogen peroxide and light (H) for Armeria linkiana, acetone and light (A) for Digitalis mariana subsp. heywoodii, and sulphuric and light (S) for Lavandula stoechas subsp. luisieri, Lavandula stoechas subsp. sampaiana and Sanguisorba hybrida. 
Hemicriptófito silicícola de distribución sudoccidental ibérica, característico de los alcornocales climácicos mesomediterráneos (Sanguisorbo - Quercetum suberis Rivas Martínez, 1987). Presenta frutos compuestos agregados, constituídos por numerosos aquenios, cada uno encerrado en un hipantio de 1-2.5 mm, oblongo y con numerosas costillas transversales (Devesa, 1987b).

La muestra estudiada, reveló tener una alta capacidad germinativa ( $84.75 \%)$ en condiciones control (C). Otras especies del género también han mostrado cierta facilidad para germinar, como Sanguisorba officinalis (Zaitseva, 1989), ampliamente utilizada por ello en la restauración de praderas naturales (MacDonald, 1993), y Sanguisorba minor, taxon para cuya germinación experimental la ISTA (1985) no estima necesario pretratamiento alguno.

La aplicación tanto de peróxido $(\mathbf{H})$ como de sulfúrico (S) propició también elevadas tasas de germinación, (78.25 \% y $92.5 \%$ ), si bien estos resultados no son estadísticamente diferentes a los del control (C). Cosa contraria ocurrió tras el tratamiento con acetona (A), ya que se observaron diferencias significativas, con reducción del porcentaje a un $50 \%$. Esto puede relacionarse con el mayor poder disolvente que este agente tiene para los compuestos orgánicos poco polares, que presumiblemente abundarán en el pericarpo del fruto.

En relación a los Indices de vigor, indicar que el tratamiento con sulfúrico (S), aceleró la velocidad de germinación de $(\mathbf{C})$ y $(\mathbf{H})$, que pasó de ser mediana a rápida, motivo por el que este agente escarificador (S) resultó ser el más conveniente para este taxon.

Respecto a la acción de la luz, resaltar la fotoblastia positiva encontrada en todos los casos, a excepción de los tratamientos con acetona. Este tipo de comportamiento, íntimamente ligado a la actividad del fitocromo, es mucho más abundante en la naturaleza que el contrario (Brabdeer, 1988).

\section{BIBLIOGRAFÍA}

ÁlVAREZ TINAUT, M.C., M. GÓMEZ y C. BLESA -1968- Influencia de diferentes pretratamientos sobre la germinación de semillas de «Lavandula spica». Pharm. Medit., 1 :7-28.
AYERBE MATEO-SAGASTA, L. y J.L CERSUELA -1982- Germinación de especies endémicas españolas. An. INIA, Serv. Forestal, 6: 17-41.

BERNAL, A.A. \& J.R. TORRES CASES -1997Seed germination of Lavandula latifolia $M$. Ecological influence and effects of gibberellic acid. Ministerio de Agricultura, Pesca y Alimentación. Madrid.

BRADBEER, J.W.-1988- Seed dormancy and germination. Blackie. New York.

CHAVAGNAT, A. - 1978- Lavender seed dormancy and germination. Acta Hort., , 83: 147-154.

COBO, M. \& A.C. ANDREU.-1988- Seed consumption and dispersal by the spur-thighed tortoise Testudo-graeca. Oikos, 51: 267-273.

DEVESA, J.A. -1987a- Lavandula. In.: B. Valdés, S. Talavera \& E. Fernández-Galiano. (eds.). Flora Vascular de Andalucía Occidental 2: 450455. Ketres. Barcelona.

DEVESA, J.A. -1987b- Sanguisorba. In: B. Valdés, S. Talavera \& E. Fernández-Galiano. (eds.). Flora Vascular de Andalucía Occidental 2: 3033. Ketres. Barcelona.

DEVESA, J.A. - 1995-Vegetación y Flora de Extremadura. Universitas. Badajoz.

GÓMEZ-CAMPO, C. -1979- The role of seed bans in the conservation of mediterranean flora. Webbia, 34: 101-107.

HUTCHINGS, M.J. \& P.J. RUSSEL -1989- The seed regeneration dynamics of an emergent salt marsh. J. Ecol., 77: 615-635.

IRIONDO, J.M., C. PÉREZ \& F. PÉREZ GARCÍA -1992- Effect of seed storage in liquid nitrogen on germination of several crop and wild species. Seed Sci. Techn., 20: 165-171.

ISTA (International Seed Test Association) - 1985The germination test. Seed Science and Technology, 13: 307-463.

JAIN, N.K. \& J.R. SAHA - 1971- Effect of storage length on seed germination in jute (Corchorus spp.). Agron. J., 63: 636-638.

JUAN RODRÍGUEZ, R. -1994-. Morfología y anatomía en frutos y semillas de Scrophulariaceae de Andalucía Occidental. Tesis Doctoral. Univ. Sevilla.

MACDONALD, A.W. - 1993- The role of seed bank and sown seeds in restoration of an english flood-meadow. J. Veg. Sci., 4: 395-400.

MAYER,A.M. \& A. POLJAKOFF-MAYBER - 1982- 
The germination of seeds. Pergamon. Oxford. MAYER, A.M. \& Y. SHAIN -1974- Control of seed germination. Ann. Rev. Pl. Physiol., 25: 167193.

NIETO FELINER; G. -1990- Armeria. In: Castroviejo \& al. (eds.). Flora Ibérica 2: 642721. CSIC. Madrid.

PAHL, M.D. -1993- Desing of a seed surface desinfection apparatus. Can. J. Pl. Sci., 73: 159-161.

PÉREZ, F., J.M. IRIONDO, M.E. GONZÁLEZ \& L.F. CARNES, J. TAPIA, C. PRIETO, R. PLAZA \& C. PÉREZ - 1995- Germination studies in endemic plant species of the Iberian Peninsula. Israel J. Bot., 43: 239-247.

RIVAS MARTÍNEZ, S. -1987- Memoria del mapa de Series de Vegetación de España. ICONA. Madrid.

RIVAS MARTÍNEZ, S., M. LOUSA, T.E. DÍAZ, F. FERNÁNDEZ-GONZÁLEZ, y J.C. COSTA. 1990- La vegetación del Sur de Portugal (Sado, Alentejo y Algarve). Itinera Geobot. 3: 5-126.

ROLDAN FERNÁNDEZ, A. -1987- Notas para una climatología de Badajoz. Instituto Nacional de Meteorología. Madrid.

ROLSTON, M. -1978- Water impermeable seed dormancy. Bot. Rev., 44: 365-396.

RUMINSKA, A. \& K. SUCHORSKA -1980Attemps at stimulation of Digitalis lanata Ehrh. germination. Herba Polon., 26: 103-110.

RUMINSKA, A., K. SUCHORSKA, T. LEWKOWICZ \& Z. KULINSA. -1977Germination of Digitalis lanata seeds under various soil conditions. Herba Polon., 23: 235245.

RYTTARI, T. \& T. LAHTI-1992-Armeria maritima ssp intermedia a rare archaeophyte in SE Finland. History, biology and conservation. Ann. Bot. Fenn., 29: 107-117.

SHCHELOKOVA, L.G. -1976- Studies on foxglove seed dormancy and germination as affected by different factors. Uch. Zap. Permsk. Gosud. Univ. Gor'kogo, 336:1 22-124.

SINGH, J.M. \& I.J SRIVASTAVA.-1990- Seed germination in lavader with acid treatment. Seed Res., 18: 86-87.

THOMPSON, P.A. -1970- Seed banks as a means of improving the quality of seed lists. Taxon, 19: 59-62.

THOMPSON, K. -1989- A comparative study of germination responses to high irradiance light. Ann. Bot., 63: 159-162.

TISCHLER, C.R., B.A. YOUNG \& M.A. SANDERSON -1994- Techniques for reducing seed dormancy in switchgrass. Seed Sci.\&Techn., 22: 19-26.

TRAN, V.N. \& A.K CAVANAGH. -1984- Structural aspects of dormancy. Seed Physiol., 2: 1-40.

WOODWARD, F.I. \& N. JONES -1984- Growth studies of selected plant species with welldefined european distributions. J. Ecol., 72: 1019-1030.

ZAITSEVA, T.A. -1989- Early stages of morphogenesis of Sanguisorba officinalis L. of different geographic origin. Rastitel'n Resursy, 25: 47-51.

Aceptado para su publicación en Abril de 1998

Dirección de los autores. Unidad de Botánica. Facultad de Ciencias. Campus Universitario. Avda. Elvas s/n. 06071 Badajoz. 